\title{
El rastreo de cáncer colorectal mediante una única sigmoidoscopía flexible reduce la mortalidad específica
}

\author{
Colorectal cancer screening with flexible sigmoidoscopy performed only once reduce specific mortality
}

\section{Objetivos}

Determinar si el rastreo del cáncer colorrectal (CCR) con una única sigmoidoscopía flexible puede reducir su incidencia y mortalidad.

\section{Diseño y lugar}

Ensayo clínico multicéntrico controlado y aleatorizado*, realizado en 14 centros del Reino Unido, con un seguimiento de 11 años.

\section{Pacientes}

Se aleatorizaron, en una proporción 2:1, 170.432 hombres y mujeres de 55 a 64 años de edad. Criterios de exclusión: incapacidad de dar su consentimiento informado, antecedente de CCR, adenomas o enfermedad inflamatoria intestinal, enfermedad grave o terminal, esperanza de vida menor a cinco años, sigmoidoscopía o colonoscopía(VCC) en los últimos tres años.

\section{Intervención}

El 71\% de los asignados al grupo intervención asistieron al rastreo donde se les realizó una sigmoidoscopía flexible con polipectomía para los pequeños pólipos y derivación para VCC
Atkin WS y col Lancet 2010; 375: 1624-1633.

si tenían criterios de alto riesgo: pólipos $\geq 1 \mathrm{~cm}$, 3 o más adenomas, adenomas tubulovellosos o vellosos en la histología, displasia severa o enfermedad maligna o 20 o más pólipos hiperplásicos sobre el recto distal. Si no se encontraban pólipos o tenían pólipos de bajo riesgo se les daba el alta.

\section{Medición de resultados principales}

Resultado principal: incidencia y mortalidad por CCR. Resultados secundarios: incidencia de cáncer distal y proximal, mortalidad por todas las causas y por causas no relacionadas con el CCR. Se analizó por intención de tratar* y por protocolo*.

\section{Resultados Principales}

En el análisis por intención de tratar la incidencia de CCR en el grupo de intervención se redujo en un $23 \%$ y la mortalidad específica en un $31 \%$ (ver tabla 1). El análisis por protocolo mostró resultados similares, con un leve beneficio adicional a favor del rastreo. Se estimó que deberían rastrearse 191 (IC95\% 145 a 277) y 489 (343 a 852) individuos para prevenir un diagnóstico adicional y una muerte por CCR, respectivamente.

Tabla 1. Incidencia y mortalidad por cáncer colorectal en el grupo intervención y control

\begin{tabular}{|c|c|c|c|c|c|}
\hline & \multicolumn{2}{|r|}{ Grupo control ( $n=112.939)$} & \multicolumn{2}{|r|}{ Grupo intervención ( $n=57.099)$} & \multirow[b]{2}{*}{ HR (IC 95\%) } \\
\hline & Casos & Tasa (100.000 personas-año, IC $95 \%$ ) & Casos & Tasa (100.000 personas-año, IC 95\%) & \\
\hline \multicolumn{6}{|l|}{ Incidencia } \\
\hline Total & 1.818 & 149 (143 a 156) & 706 & 114 (106 a 123) & $0,77(0,70$ a 0,84$)$ \\
\hline Distal & 1.192 & 98 (92 a 103) & 386 & $62(57$ a 69$)$ & $0,64(0,57$ a 0,72 \\
\hline Proximal & 628 & $51(48$ a 56$)$ & 311 & $50(45$ a 56$)$ & $0,98(0,85$ a 1,12 \\
\hline \multicolumn{6}{|l|}{ Mortalidad } \\
\hline CCR & 538 & $44(40$ a 48) & 189 & $30(26$ a 35$)$ & $0,69(0,59$ a 0,82$)$ \\
\hline Otras causas & 13.230 & $1.080(1.062$ a 1.099$)$ & 6.586 & $1.062(1.037$ a 1.088$)$ & $0,98(0,95$ a 1,01$)$ \\
\hline
\end{tabular}

\section{Conclusiones}

La sigmoidoscopía flexible es una prueba segura y práctica que cuando es ofrecida por única vez entre los 55 y 64 produce un Fuente de financiamiento: Medical Research Council, National Health Service beneficio sustancial y duradero.

\section{Comentario}

El CCR es la segunda causa de muerte por cáncer. Dos tercios de los casos se localizan en el colon distal y la edad es uno de los factores de riesgo más importantes, ya que el $90 \%$ se diagnostica en pacientes mayores de 50 años.

Las medidas de rastreo actualmente disponibles son: sangre oculta en materia fecal (SOMF), colon por enema, sigmoidoscopía, videocolonoscoía (VCC) y colonoscopía virtual por tomografía computada. La SOMF mediante la prueba del guayaco ha demostrado reducir la mortalidad pero la adherencia es baja y cualquier resultado positivo es indicación de realizar una VCC para determinar el origen del sangrado. La sensibilidad reportada de la VCC es del $98 \%$ para lesiones mayores o iguales a $6 \mathrm{~mm}$. Al compararla con la sigmoidoscopía hay mas posibilidades de complicaciones (perforación y sangrado, en 1 por cada 1.000 procedimientos), necesita una preparación rigurosa y sedación.

Este estudio demuestra que el rastreo con sigmoidoscopía entre los 55 a 64 años disminuye la incidencia y mortalidad del CCR distal, mientras que no tuvo efecto en la incidencia a nivel proximal. Remarcan las ventajas de la sigmoidoscopía ya que puede ser realizada por personal de salud entrenado (médicos generalistas, enfermeras, técnicos), la duración de 5 a 15 minutos, no necesita sedación y luego del estudio el paciente continúa con su actividad habitual. Se realizaron cuestionarios post intervención para evaluar la experiencia de cada participante, el $80 \%$ respondió que no tuvo dolor o fue leve y solo el $3 \%$ lo describió como severo. En general la aceptabilidad fue alta.

\section{Conclusiones del comentador}

Resulta claro que necesitamos revisar los métodos de rastreo que utilizamos en la práctica diaria ya que la SOMF tiene baja adherencia y la VCC, aunque es el gold standard, es impracticable como método de rastreo para la población general por su bajo acceso. Lo ideal sería una política de rastreo nacional donde pudiéramos llegar a toda la población con un método que tenga buena adherencia, sea costo-efectivo y el mejor en disminuir la incidencia y mortalidad por CCR.

María de las Nieves Ganiele [ Servicio de Medicina Familiar y Comunitaria del Hospital Italiano de Buenos Aires.

*Ver glosario maria.ganiele@ hospitalitaliano.org.ar ]

Ganiele M. El rastreo de cáncer colorectal mediante una única sigmoidoscopía flexible reduce la mortalidad específica. Evid Act Práct Ambul. Jul-Set 2012;15(3):90. Comentado de: Atkin WS, Edwards R, Kralj-Hans I. Once-only flexible sigmoidoscopy screening in prevention of colorectal cancer: a multicentre randomised controlled trial. Lancet 2010; 375:1624-1633. PMID: 20430429.

Bibliografía

1.Fletcher RH. Test for screening for colorectal cancer: stool tests, radiologic imaging and endoscopy. En: UpToDate, Basow, DS (Ed), UpToDate, Waltham, MA, 2012. 